Voix et Images

volxetimages

\title{
Pour petits, ados et adultes avertis
}

\section{Lucie Robert}

Volume 18, numéro 3 (54), printemps 1993

Littérature, folie, altérité

URI : https://id.erudit.org/iderudit/201059ar

DOI : https://doi.org/10.7202/201059ar

Aller au sommaire du numéro

Éditeur(s)

Université du Québec à Montréal

ISSN

0318-9201 (imprimé)

1705-933X (numérique)

Découvrir la revue

Citer cet article

Robert, L. (1993). Pour petits, ados et adultes avertis. Voix et Images, 18(3), 629-635. https://doi.org/10.7202/201059ar d'utilisation que vous pouvez consulter en ligne.

https://apropos.erudit.org/fr/usagers/politique-dutilisation/ 


\section{Dramaturgie}

\section{Pour petits, ados et adultes avertis}

\section{Lucie Robert, Université du Québec à Montréal}

L'écriture destinée aux enfants et aux adolescents n'occupe dans la littérature qu'une place marginale, au point où l'on se demande parfois s'il arrivera un jour qu'une revue universitaire - pourquoi pas Voix et Images? - accorde quelqu'importance à ce qui reste un des secteurs les plus populaires du marché du livre au Québec. Il n'en est toutefois plus exactement ainsi du théâtre et, parmi les praticiens qui consacrent l'essentiel de leur travail à ces jeunes publics, certains ont atteint des renommées que les autres envient à juste titre. Le théâtre pour jeunes publics a en effet conquis ses lettres de créance, sinon ses lettres de noblesse, et il montre maintenant des signatures reconnues et recherchées. Dans la quatrième et plus récente livraison de Veilleurs de nuit ${ }^{1}$, Hélène Beauchamp qui, depuis plusieurs années, consacre l'essentiel de sa carrière à étudier les productions théâtrales pour jeunes publics, écrit que les "artistes du théâtre jeunes publics signent désormais leurs œuvres: leurs styles sont repérables* et que "vingt ans de travail, d'exposition publique, de tournées à l'étranger mais aussi de luttes sur leur propre territoire pour gagner le respect de leurs pairs et de la critique, donnent désormais à reconnaître des auteurs, des metteurs en scène, des acteurs, des compagnies. (p. 56).

Parmi ces signatures, du moins en ce qui concerne l'aspect dramaturgique de ce théâtre, domine nettement et toujours celle de LouisDominique Lavigne, qui vient de publier trois nouveaux textes en un peu plus d'un an, un pour les enfants, Les Petits Orteils ${ }^{2}$, et deux pour adolescents, Le Sous-sol des anges ${ }^{3}$ et Tu peux toujours danser ${ }^{4}$.

Je dois reconnaître avoir été séduite par la lecture des Petits Orteils qui montre toute la maîtrise que Lavigne a acquise dans cet art particulier. En effet, il n'y a pas d'écriture plus difficilement littéraire que celle du théâtre pour enfants, en particulier, comme c'est le cas ici, pour les tout petits. La littérature n'existe que par un certain travail sur la langue et une dramaturgie donnée à lire n'offre de plaisir que si elle manifeste cette dimension littéraire; c'est ce qui explique que certains textes dramatiques, si intéressants à la scène, ne soient que des pensums de lecture. Dans le théâtre pour enfants, l'accent est généralement mis sur la dimension visuelle du spectacle, l'attention 
étant généralement captée par les couleurs et le mouvement. Les textes antérieurs de Lavigne présentaient cette caractéristique, c'est-àdire une incomplétude dans l'écriture, comblée en partie par le "Cahier d'activités" que l'on ajoute à l'édition du texte dramatique luimême.

Produite par le Théâtre de Quartier et créée au Centre national des arts à Ottawa le 30 septembre 1991, dans une mise en scène de Lise Gionet, Les Petits Orteils est une pièce de 45 minutes, écrite pour deux comédiens et destinée aux enfants d'âge préscolaire. Divisée en neuf tableaux, la pièce cherche à représenter les difficultés et l'exaltation que suscite l'arrivée d'un second enfant dans une famille. Deux comédiens jouent tous les rôles et se transforment à l'aide de quelques accessoires. Dans la préface de la pièce, Louis-Dominique Lavigne précise avoir cherché à s'éloigner de certaines émissions de télévision qui proposent * un spectacle modulaire où il n'y aurait pas d'histoire et où s'enchaîneraient toutes sortes de situations qui illustreraient différentes problématiques de la petite enfance. (p. 7). On pense à PassePartout que produit Radio-Québec. Lavigne, au contraire, se plie "aux exigences de la fable. Il veut raconter "de belles histoires", pour " intéresser les tout-petits et tous les adultes qui ont encore un peu d'enfance en eux * (ibid.). Il y parvient, - Mathilde et son grand-père séduisent facilement -, mais là n'est pas l'essentiel de son travail, qui réside plutôt dans cette sorte de fête à laquelle est convoqué le langage et qui fait que, d'un côté, certaines expressions trouvent un sens propre plus figuré qu'on aurait cru possible ("Mathilde prend la main de grand-papa. La main se détache" [p. 18]) ou qui, d'un autre côté, réinscrit certaines répliques dans des chansons connues $(*$ Ding! Dong! Ah! vous dirais-je vraiment / qui est-ce qui sonne en avant. / Moi je dis que ça résonne / comme une grande personne.. [p. 20]) Aussi la mise en scène peut-elle recréer au besoin ce fonctionnement modulaire qui correspondrait mieux à la capacité d'écoute des enfants, sans jamais rompre la fable.

Par comparaison, Le Pain de la boucbe ${ }^{5}$, de Joël da Silva, produite également par le Théâtre de Quartier, mais créée le 9 décembre 1992 à Lac Mégantic et mise en scène par l'auteur avec la collaboration de Lise Gionet et Louis-Dominique Lavigne (ce dernier étant également un des interprètes), n'offre pas les mêmes qualités à la lecture. Da Silva, qui a déjà publié une première pièce, La Nuit blanche de Barbebleue, en 1989, et qui a conçu la trame musicale des Petits Orteils, propose ici le livret d'un spectacle de thêâtre musical. Librement adapté du conte de Grimm, Hansel et Gretel, — "Hansel et Gretel sont maintenant octogénaires et se portent très bien merci* (p. 13) -, ce 
livret, intéressant par ailleurs, présente une suite de dialogues dont l'écriture ne laisse pas entendre la musique. Aussi ne peut-on pas tirer tout ce qu'il est possible du texte par la seule lecture et le projet énoncé dans la "Présentation * - "Le Pain de la boucbe [...] demeure aussi un texte qui se lit, comme se lit une belle histoire, un conte ou un poème. (p. 11) — n'est guère réalisé.

Encore par comparaison, le Petit Monstre 6 de Jasmine Dubé, créé par le Théâtre Bouches décousues, le 24 mai 1992, au Centre culturel Strathearn, dans une mise en scène de Claude Poissant assisté de Mathieu Marcil, se présente dans les formes plus classiques de la dramaturgie. L'accent est mis sur le dialogue - on n'imagine pas pièce plus bavarde! - et sur la représentation pure, c'est-à-dire sur le " donner à voir et à entendre . Jasmine Dubé avait pour projet "d'écrire une pièce qui s'adresserait aux enfants et à leurs adultes et qui mettrait en relief une relation de tendresse entre un père et un fils" (p. 7). Le sujet est intéressant et l'auteure parvient assez bien à faire ressortir l'affection qui unit le père et le fils, seuls en scène avec quelques personnages-accessoires. Le propos est cependant un peu mince et la "monstruosité du petit garçon se révèle à la longue bien "raisonnable „, à peine de quoi s'énerver un peu, rien même pour se fâcher. L'interaction avec le public est limitée et ne laisse comme possible que le voyeurisme, effet sans doute entraîné par la présence d'un double public, inégalement intéressé au propos, et ayant une relation fort différente au spectacle. Jasmine Dubé s'est retrouvée piégée par le personnage du père qui, une fois réveillé (c'est l'objet de la pièce et le projet du petit monstre), devient lui-même raisonnable. On voit mal ce qui, dans ce texte, peut intéresser les enfants.

\section{$* *$}

On peut dégager trois conceptions du théâtre pour les adolescents. Selon la première, qui était très en vogue dans mes jeunes années et qui a perdu beaucoup de popularité depuis, il faut laisser les adolescents faire leur propre théâtre (à l'école, au cégep, dans la rue), conception tributaire des programmes d'enseignement du français au secondaire et des programmes d'emploi qui, dans ces annéeslà, ont largement contribué à la naissance du jeune thêâtre. Une deuxième conception voit dans les adolescents le public de demain, qu'il faut mettre au fait et à qui il faut présenter les classiques. Cette vision scolaire, vaguement élitiste, est toujours celle de la Nouvelle Compagnie théâtrale, laquelle accumule les échecs et les mauvaises 
productions, en plus de susciter les protestations continues des critiques, en tout premier lieu celles d'Hélène Beauchamp qui constate, à ce propos, que servir aux adolescents "de l'hier réchauffé, c'est les nourrir de bien piètre façon ${ }^{7}$. La troisième conception est issue du thêâtre didactique et elle a pour projet de faire comprendre et réfléchir tout en divertissant. Efficace, semble-t-il, dans son projet, cette dernière conception n'a cependant aucune chance de donner lieu à une dramaturgie qui manifeste un quelconque sens de l'humour.

Louis-Dominique Lavigne a publié récemment deux pièces de ce genre: Le Sous-sol des anges, produite par le Théâtre de Carton et créée le 8 novembre 1984, dans une mise en scène de Lorraine Pintal; et Tu peux toujours danser, produite par le théâtre le Clou et créée le 16 octobre 1990, dans une mise en scène de Claude Poissant. La première traite du suicide chez les jeunes et elle est construite de manière à faire reconnaître les signes avant-coureurs et les solutions au problème. Elle est d'ailleurs suivie d'un guide d'accompagnement réalisé par Suicide-Action Montréal Inc. La seconde, dont le titre rappelle un vers de la "La cigale et la fourmi", parle des maladies transmises sexuellement, en particulier du sida, en présentant plusieurs scénarios de contagion et en montrant explicitement la façon de se protéger. Les deux pièces sont construites de manière semblable: il s'agit de "dramatiser " un problème de société et de faire suivre la représentation d'une discussion. Aussi le texte ne peut-il qu'être un peu figé, ne laissant que peu de place à l'invention. Le travail d'uécrivain scénique", pour reprendre l'expression que Louis-Dominique Lavigne utilise pour désigner ce travail, est ainsi plus technique que véritablement artistique: il faut présenter la situation "correctement», faute de quoi le message sera incomplet ou imprécis. À vrai dire, même quand le travail est bien mené - ce qui est le cas ici —, le théâtre didactique pour adolescents n'offre pas la moitié de l'intérêt littéraire qu'offre le théâtre pour enfants.

À quoi ressemblerait une pièce pour adolescents qui ne serait pas entièrement soumise à une conception didactique? À Pied de poule doit-on reconnaître, tout en mesurant l'horreur d'une telle réponse. Précisons. D'un côté, le texte que Marc Drouin vient tout juste de publier, plus de dix ans après sa création et, écrit-il dans sa présentation, à la demande des jeunes, agit comme un miroir renvoyant au public une image idéale de lui-même. Les personnages sont des 
adolescents qui produisent des biens culturels de consommation propres à ce groupe d'âge: musique pop-rock, film d'aventures, héros à la James Dean, adultes méchants et/ou crétins. De l'autre côté, il sombre parfois dans une telle facilité que l'on ne peut s'empêcher de $s^{\prime}$ inquiéter pour l'avenir de cette génération. Je n'ai pas vu le spectacle à la scène (ni lors de la création en 1982, ni à la reprise de l'été 1991), mais j'ai en mémoire à la fois une version enregistrée sur disque et une version télévisuelle, présentée à Radio-Canada, aux Beaux Dimanches, il me semble. J'ai en fait un assez bon souvenir de la musique (de Robert Léger): et un meilleur encore des deux interprètes du rôle de Dolbie Stéréo, assez en tout cas pour n'avoir pas vraiment remarqué le tissu de clichés qui composent le texte du spectacle ("Un p'tit canapé, François? Nous ne nous étendrons pas sur le sujet», [p. 25]). Il faut dire que la version sur disque ne comprenait que les chansons et que la version télévisuelle était considérablement écourtée.

L'anecdote est simple et classique: après avoir fait de François Tremblay, dit François Perdu, l'idole de toute une jeunesse, un producteur de cinéma, Desmond Bigras, cherche à le tuer pour s'en débarrasser, empocher les bénéfices et séduire la jeune première, Olive Houde. La suite n'est pas mal: Bigras n'a guère prévu la chance inouie qui colle aux fesses du jeune premier qui évite de justesse tous les pièges et revient à Montréal le jour de son supposé enterrement (qui est aussi celui de la première du film) dévoiler lé complot. La fin est un cliché: en tirant sur François, les policiers, qui croient arrêter un imposteur, atteignent Olive Houde qui rend l'âme aussitôt; Desmond Bigras se suicide devant la foule qui attend son idole et François Perdu entreprend de raconter son aventure. C'est le genre de fin qu'utilisent les auteurs qui ne savent comment sortir de leur histoire et qui font en sorte que leurs personnages s'en tirent tout seuls, comme par miracle.

J'aurais dû me contenter du souvenir pas si mauvais qui me reste d'une production musicale du genre de celles qu'a popularisées le tandem Luc Plamondon-Michel Berger, c'est-à-dire Starmania ou, plus récemment, La Légende de Jimmy. La qualité des interprètes permettait alors de transporter un texte qui à la lecture s'avère vide de sens et d'intérêt, une accumulation des clichés les plus faciles empruntés au théâtre, à la musique, mais surtout à la publicité, avec un étonnant personnage qui a pour nom... Françoise Gaillard, et qui amène à penser que l'auteur du livret n'est pourtant pas exactement un analphabète. On trouve d'ailleurs maintes références implicites à d'autres œuvres littéraires, cinématographiques ou télévisuelles, comme celle-ci, citation indirecte empruntée au Sol de Marc Favreau: "[...] disons qu'au début ses études furent très primaires et que par la suite elles devinrent très 
secondaire * (p. 23) ou encore, cette référence parodique: « c'est Denis Archange, réalisateur du fameux film Le Regain de l'empire amérindien. (p. 34). Aucune de ces références n'est assez populaire pour être vraiment reçue par un public adolescent, fût-il cultivé. Nous sommes dans l'ordre d'une esthétique hybride, née à la fois du vidéo-clip, où la fable ne sert qu'à l'enchaînement des tableaux musicaux, et de la publicité, où le message est d'abord référentiel plutôt qu'informatif, c'est-à-dire que, faute de temps ou d'espace pour livrer une certaine information, on se contente de référer à un autre texte qui la contient ou la suggère. Sauf qu'ici, ce n'est pas par manque de temps ou d'espace.

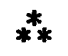

À propos d'esthétique publicitaire, je voudrais signaler la parution des actes du colloque "Claude Meunier, texte et représentation", que Gabrielle Pascal et André Smith ont organisé à l'université McGill en septembre 1990. Sous le titre un peu étonnant de Claude Meunier, dramaturge ${ }^{9}$, se trouvent réunis des articles d'universitaires et de praticiens qui tentent de circonscrire la contribution particulière de Meunier à l'humour québécois et de situer cet humour dans l'histoire. C'est l'appellation de "dramaturge" qui étonne ici puisque qu'elle nous oriente vers une activité théâtrale somme toute marginale dans le travail de Meunier, qui n'a publié à ce jour que deux pièces écrites en collaboration avec Louis Saia, Les Voisins et Appelez-mot Stéphane, et dont les autres productions n'existent que sur disque (si vous trouvez les vieux enregistrements de Paul et Paul), à la télévision (les publicités de Pepsi en particulier), au cinéma (depuis le tournage de Ding et Dong. Le film) ou sur scène (Broue - n'avait-il pas écrit aussi un des textes de Mousse? - et La P'tite Vie).

En fait, la contribution la plus importante de Meunier à l'histoire du spectacle n'existe que dans la mémoire de son public et, à moins d'une intervention éditoriale - le plus vite serait le mieux - , elle est ainsi vouée à être oubliée aussi rapidement que ne l'avait été à l'époque le burlesque (que reste-t-il des Olivier Guimond, père et fils?), tradition à laquelle Chantal Hébert rattache cette forme d'humour. Outre l'article de Chantal Hébert, on lira notamment ceux de Michèle Nevert sur les publicités de Pepsi, de Diane Desrosiers-Bonin sur le carnavalesque, de Stéphane Lépine, qui vient jeter une rafraîchissante note discordante dans ce qui autrement reste un concert d'éloges, et du publicitaire Robert Thibodeau, qui prouve (enfin!) qu'on peut parler intelligemment du travail d'un collaborateur. Regrettons cependant que la formule choisie par les organisateurs du colloque, et qu'ils avaient déjà 
mise en place lors du colloque sur Marie Laberge ${ }^{10}$, nous oblige à digérer du même coup les propos profondément narcissiques de certains autres collaborateurs de Meunier, en particulier ceux du producteur Roger Frappier ( (Claude Meunier, le cinéma et moi ), propos dont je me serais passé avec le plus grand bonheur.

1. Veilleurs de nuit 4. Bilan de la saison thêâtrale 1991-1992, sous la direction de Gilbert David, Montréal, Les Herbes rouges, 1992, 303 p. Le texte d'Hélène Beauchamp, -Jeunes publics. Désormais, des signatures. est en p. 56-63.

2. Louis-Dominique Lavigne, Les Petits Orteils. Théâtre, Montréal, VLB éditeur, coll. -Théâtre pour enfants , 1991, $81 \mathrm{p}$.

3. Id., Le Sous-sol des anges. Jeune théâtre guide d'accompagnement réalisé par Suicide-Action Montréal Inc., texte de Monique Séguin et Réjean Marier, Montréal, VLB éditeur, 1991, $153 \mathrm{p}$.

4. Id., Tu peux toujours danser. Jeune thêâtre, Montréal, VLB éditeur, 1991, $109 \mathrm{p}$.

5. Joël da Silva, Le Pain de la boucbe. Théâtre, Montréal, VLB éditeur, coll. "Théâtre pour enfants :, 1993, 103 p.

6. Jasmine Dubé, Petit Monstre, Montréal, Leméac, coll. .Théâtre jeunesse ", 1993, 64 p.

7. Hélène Beauchamp, loc. cit., p. 61.

8. Marc Drouin, Pied de poule. Thêâtre, Montréal, VLB éditeur, 1992, 146 p.

9. Gabrielle Pascal et André Smith (directeurs) Claude Meunier, dramaturge. Actes du colloque "Claude Meunier, texcte et représentation, Montréal, VLB éditeur, 1992, 138 p.

10. Gabrielle Pascal et Andre Smith (directeurs), Marie Laberge, dramaturge. Actes du colloque international, Montréal, VLB éditeur, 1989, $145 \mathrm{p}$. 